\title{
The Traumatic Experience of Clinical Nurses During the COVID-19 Pandemic: Which Factors are Related to Post-Traumatic Growth?
}

\author{
Xin Tong Zhang* \\ Song Song Shi* \\ Yu Qin Ren \\ Li Wang
}

Department of Emergency, Nantong First People's Hospital, Nantong, 22600I, People's Republic of China

*These authors contributed equally to this work
Correspondence: Yu Qin Ren

Department of Emergency, Nantong First People's Hospital, No. 6 of Haierxiang

North Road, Chongchuan District,

Nantong, 22600I, People's Republic of

China

Tel +86 I5962956088

Email renyuqindr@2Icn.com
Objective: Post-traumatic growth (PTG) is a positive result of fighting against traumatic events. This study aimed to investigate the current status of PTG of clinical nurses and analyze its influencing factors.

Methods: A total of 1790 nurses participated in the study and completed the questionnaire. Demographic data and related scales of PTG, post-traumatic stress disorder, coping style, social support, and self-efficacy were collected online. Through univariate analysis and multiple linear regression analysis, the related influencing factors were studied.

Results: The total score of PTG of 1790 nurses was $67.17 \pm 14.79$. The analysis revealed that good social support and self-efficacy were important factors to improve the level of PTG of clinical nurses, while bad psychological state and working for many years were the negative factors of PTG.

Conclusion: Good social support and self-efficacy can help clinical nurses cope with the novel coronavirus disease 2019 pandemic and accept the disease's challenges. If these factors can be considered in clinical practice, this will help promote clinical nurses' mental health. Keywords: COVID-19, post-traumatic growth, psychological impact, nurse, influencing factors

\section{Introduction}

In December 2019, the new coronavirus pneumonia (novel coronavirus disease 2019, COVID-19) first broke out in Wuhan, a major transportation hub in central China. ${ }^{1}$ It spread rapidly across China and then the whole world. COVID-19 has become a major pandemic that is threatening the lives of people around the world. At present, the number of COVID-19 infections has exceeded 10 million. $^{2}$ The outbreak of infectious disease often results in a series of psychological problems. ${ }^{3}$ As the frontline of epidemic or pandemic prevention, clinical nurses not only undertake the heavy burden of saving lives and fighting the pandemic, but they also face the huge risks and pressures of infection from the virus. Due to the influence of multiple stressors, clinical nurses are prone to various psychological problems. The negative emotions of clinical nurses, such as anxiety, depression, fear, and post-traumatic stress disorder (PTSD symptoms), in addition to ways to improve their mental health, have increasingly become the subject of intensive research.,

Post-traumatic growth (PTG) is considered a positive result of fighting against traumatic events. It is defined as "positive psychological change experienced as 
a result of the struggle with highly challenging life circumstances". 6 PTG is mainly reflected in the individual's profound understanding of the value of life after experiencing a traumatic event. ${ }^{7}$ Therefore, they may reset the order of important matters in life, enhance their personal abilities, and get closer to others. ${ }^{8}$ Some studies suggested that PTG can improve the quality of life and mental state of people who experience traumatic events. ${ }^{9,10}$ Among those who have experienced traumatic events, the PTG of cancer patients has always been the focus of interest. $^{11,12}$ The factors of their PTG mainly relate to social support, the time and degree of illness, and coping style. $^{12-14}$ There has been little recent progress in identifying the influencing factors of PTG among people who fight against major infectious diseases, especially medical workers.

Therefore, this study investigates the current status of PTG of clinical nurses fighting against the COVID-19 pandemic and analyzing the influencing factors, and we hypothesized that good social support and self-efficacy can help clinical nurses cope with the novel coronavirus disease 2019 pandemic and accept the disease's challenges. Our findings will provide a relevant reference for nursing managers to improve clinical nurses' PTG and further promote their mental health.

\section{Materials and Methods}

\section{Setting and Participants}

The study is descriptive and was conducted during the period when the COVID-19 pandemic was stable The questionnaires were distributed and collected over four days (from June 6 to June 9, 2020). Participants were clinical first-line nurses who were involved in screening people with suspected COVID-19, and nursing patients with suspected and confirmed COVID-19 during the COVID-19 pandemic. A total of 1790 nurses participated in the study and completed the questionnaire. Since this survey was carried out during the COVID-19 pandemic, to reduce face-to-face communication and contact, we chose to use a mobile app to construct an anonymous questionnaire, which was distributed to participants via WeChat after obtaining their informed consent.

\section{Demographic Data and Questionnaire}

Demographic data included gender, age, marital status, educational background, department, professional title, and whether the participants had children.
The Post-Traumatic Growth Inventory (PTGI) was developed by Tedeschi and Calhoun in $1996^{15}$ and is mainly used to assess individuals' psychological changes after trauma. The scale includes five dimensions: relating to others, new possibilities, personal strength, spirituality, and appreciation of life. A total of 21 items are used. Each item is scored on a 6-point Likert scale. The total score range of the scale is $0-105$ points, and the higher the score, the higher the individual's level of growth after trauma. The Cronbach's alpha coefficient of this scale is 0.90 , which has good reliability and validity. ${ }^{16}$

The Post-Traumatic Stress Disorder CheckList-Civilian Version (PCL-C) was compiled by the United States PTSD symptoms Research Center in $1994^{17}$ and was introduced into China by Chao Jiang's team. ${ }^{18}$ The Cronbach's alpha coefficient of the Chinese version of the scale is 0.88-0.94, and the retest reliability is $0.83-0.88 .{ }^{19}$ There are 17 items in this scale, which are mainly used to evaluate the three main symptom groups of PTSD symptoms: the recurring traumatic experience symptom group, persistent avoidance symptom group, and persistent anxiety and increased alertness symptom group. Items are scored on a 5-point Likert scale, with a total score of $17-85$. The higher the score, the greater the likelihood of patients suffering from PTSD symptoms. In the Chinese version of the PCL-C, a total score of $\geq 38$ is positive for PTSD symptoms.

The Simplified Coping Style Questionnaire (SCSQ), which was compiled by Xie Yaning's team, is divided into two dimensions and includes 20 items. $^{20}$ Questions 1-12 are related to positive coping dimensions, and the subsequent questions are related to negative coping dimensions. The SCSQ is a self-assessment questionnaire that is scored using a multi-level scoring method. Each question is described by four options (not adopted, occasionally adopted, sometimes adopted, and frequently adopted), and the corresponding scores are $0,1,2$, and 3 .

The Social Support Rate Scale (SSRS) was compiled by Xiao Shuiyuan and Yang Desen. ${ }^{21}$ The Cronbach's alpha coefficient of this scale is 0.89-0.94, and the retest reliability is 0.92 . The SSRS includes three objective support items, four subjective support items, and three social support utilization items. Items $1-4$ and $8-10$ are singlechoice items, with 1-4 points respectively. Item 5 has five options, and the total score is recorded. The score for each option ranges from 1 to 4 points, ranging from "none" to "full support." In items 6 and 7, if participants answer "no source," they will score 0 points, and if they answer "the following sources," they will accumulate scores based on 
the number of options they choose. The scale is divided into low-level social support ( $\geq 22$ points), medium-level social support (23-44 points), and high-level social support (45-66 points) according to the total score.

The General Self-Efficacy Scale (GSES) was compiled by the German health psychologist Professor Ralf Schwarzer and his colleagues in $1981 .^{22}$ The Chinese version was translated into Chinese by Zhang Jianxin's team. ${ }^{23}$ The scale has 10 questions, each of which is scored by a 4-point Likert scale - the lower the score, the lower the self-efficacy. The score of the scale is divided into three levels: $<20$ points, low self-efficacy level; 20-30 points, medium self-efficacy level; and $>30$ points, high self-efficacy level. The scale has good reliability and validity after measurement.

\section{Statistical Analysis}

In this study, PTG and the comparison of differences in various dimensions were analyzed using analysis of variance and the $t$-test. Sample size was calculated based on previous study results of PTG among medical personnel. Normality was tested by Shapiro-Wilk test. Descriptive statistics were used to analyze the clinical first-line nurses' demographic and clinical data, PTG, PTSD symptoms, social support, self-efficacy, and coping style. The count data were described by frequency and percentage, and the measurement data were described by mean and standard deviation (SD). Pearson correlation analysis was used to explore the correlation between nurses' PTG and PTSD symptoms, general self-efficacy, social support, and coping style. Finally, taking the score of PTGI as the dependent variable and all the other indicators as the independent variables, a multivariate linear stepwise regression analysis was performed to identify the main influencing factors of clinical first-line nurses' PTG. Data with a value of $\mathrm{p}<0.05$ were considered statistically significant. Analyses were performed using IBM SPSS version 20.0 (IBM Co. Ltd, Chicago, IL, USA).

\section{Results}

\section{Demographic and Clinical Data}

A total of 1790 individuals were enrolled in this study. Results showed a statistical difference in nurses' academic qualifications and professional titles. Significant difference was found in education level and professional title.

Through post-hoc analysis, it was found that nurses with a bachelor's degree had a higher PTGI score than those with a college degree. We also found that with the increase in professional title, the PTGI score decreased.

\section{Psychological Assessment}

The mean PTGI total score of the 1790 nurses in this study was 67.17 (SD 14.79). Among the five dimensions, the "relating to others" dimension scored the highest, and the "spirituality" dimension scored the lowest (see Table 1). The nurses' self-efficacy and social support were at a medium level, with average scores of $26.69 \pm 5.50$ and $41.50 \pm 8.47$, respectively. Their PTSD symptoms scores were higher, but most of the nurses adopt a positive coping style when facing difficulties.

\section{Correlations}

Pearson correlation analysis showed that during the COVID-19 pandemic, clinical first-line nurses' PTG was positively correlated with coping style, social support, and self-efficacy and was negatively correlated with PTSD symptoms (see Table 2).

\section{Multiple Regressions}

Taking the total score of PTGI as the dependent variable, the variables with statistical significance in single factor analysis and the scores of PTSD symptoms, general selfefficacy, social support, and coping style were selected as independent variables, and multiple regression analysis was performed. Independent variable assignment criteria were education (technical secondary school degree $=1$; college degree $=2$; bachelor's degree $=3$; master's degree or higher $=4$ ) and professional title (nurse $=1$; senior nurse $=2$; nurse-in-charge $=3$; deputy chief nurse $=4$; chief nurse $=5$ ), and the total score of each scale was inputted according to the original value. The results showed that professional title, PTSD symptoms, general self-efficacy, social support, and coping style were the factors that are related to the PTG level of nurses during the COVID-19 pandemic (see Table 3).

\section{Discussion}

\section{Positive Outcomes of PTG}

The PTGI score of 1790 clinical first-line nurses in this survey is $67.17 \pm 14.79$, indicating that the nurses in this survey have a certain level of PTG. The results of this study are higher than those of domestic scholars such as Ma Xiaojiao ${ }^{24}$ regarding the PTG of emergency nurses. The factors of PTG mainly relate to social support, the 
Table I Demographic Data and First-Line Clinical Nurses' PTG Score ( $\mathrm{N}=1790)$

\begin{tabular}{|c|c|c|c|c|}
\hline & $\mathbf{N}(\%)$ & PTGI Score & F/t value & $P$ value \\
\hline Age (Years) & & & $F=2.705$ & 0.067 \\
\hline$\leq 30$ & $809(45.20)$ & $67.76 \pm 15.06$ & & \\
\hline $31 \sim 40$ & 675 (37.70) & $66.12 \pm|4.6|$ & & \\
\hline$\geq 41$ & $306(17.10)$ & $67.89 \pm 14.36$ & & \\
\hline Gender & & & $t=-1.060$ & 0.289 \\
\hline Female & $1779(99.40)$ & $67.19 \pm 14.81$ & & \\
\hline Male & II $(0.60)$ & $62.45 \pm 11.55$ & & \\
\hline Marriage & & & $F=0.276$ & 0.759 \\
\hline Unmarried & $404(22.60)$ & $67.41 \pm 16.15$ & & \\
\hline Married & I365 (76.20) & $67.06 \pm 14.37$ & & \\
\hline Divorce or others & $21(1.20)$ & $69.14 \pm 15.13$ & & \\
\hline Education & & & $F=3.780$ & 0.010 \\
\hline Technical secondary school degree & $15(0.80)$ & $66.47 \pm 19.29$ & & \\
\hline College degree & $341(19.10)$ & $65.18 \pm 15.69$ & & \\
\hline Bachelor & I 427 (79.70) & $67.59 \pm 14.47$ & & \\
\hline Master or higher degree & $7(0.40)$ & $78.29 \pm|6.5|$ & & \\
\hline Department & & & $F=1.027$ & 0.400 \\
\hline Emergency department & $131(7.30)$ & $66.52 \pm 16.52$ & & \\
\hline Pre-examination triage & $96(5.40)$ & $69.18 \pm 15.65$ & & \\
\hline Fever clinics & $66(3.70)$ & $69.09 \pm 13.66$ & & \\
\hline Department of Infectious Diseases & $34(1.90)$ & $65.18 \pm 17.42$ & & \\
\hline Traffic checkpoint or medical observation point & $29(1.60)$ & $70.14 \pm 14.47$ & & \\
\hline Other clinical departments & $1434(80.10)$ & $66.99 \pm 14.55$ & & \\
\hline Professional title & & & $F=6.285$ & $<0.001$ \\
\hline Nurse & $315(17.60)$ & $69.80 \pm 14.89$ & & \\
\hline Senior nurse & II $45(64.00)$ & $66.13 \pm 14.60$ & & \\
\hline Nurse-in-charge & $125(7.00)$ & $65.50 \pm|4.9|$ & & \\
\hline Deputy chief nurse & $178(9.90)$ & $69.58 \pm 14.85$ & & \\
\hline Chief nurse & $27(1.50)$ & $72.00 \pm 15.15$ & & \\
\hline With or without children & & & $F=1.037$ & 0.375 \\
\hline 0 & 544 (30.39) & $67.16 \pm 15.75$ & & \\
\hline 1 & $1033(57.7 \mathrm{I})$ & $66.95 \pm \mid 4.21$ & & \\
\hline 2 & 209 (11.68) & $67.25 \pm 14.68$ & & \\
\hline$\geq 3$ & $4(0.22)$ & $78.00 \pm 14.44$ & & \\
\hline Years of working & & & $F=2.624$ & 0.073 \\
\hline$\leq 5$ & $443(24.70)$ & $68.34 \pm 15.32$ & & \\
\hline $6 \sim 10$ & $628(35.10)$ & $66.25 \pm 14.50$ & & \\
\hline$\geq 11$ & $719(40.20)$ & $60.92 \pm 9.16$ & & \\
\hline
\end{tabular}

Note: There was statistical significance between the junior college group and the undergraduate group, between the nurse group and the nurse nurse group, and between the nurse group and the supervisor nurse group.

time and degree of illness, and coping style. ${ }^{12-14}$ There has been little recent progress in identifying the influencing factors of PTG among people who fight against major infectious diseases, especially medical workers.

Tedeschi and Calhoun ${ }^{15}$ believe that social support is a direct predictor of PTG. Survivors with a high level of social support were more likely to receive more emotional or material support from family members, friends, social groups, and colleagues through sharing and communication. ${ }^{14,25}$ Our study showed that the total PTG score of nurses was significantly positively correlated with the total social support score, with subjective support 
Table 2 Pearson Correlations Between PTG, Coping Style, Social Support, Self-Efficacy and PTSD (N = 1790)

\begin{tabular}{|c|c|c|c|c|c|c|c|}
\hline \multicolumn{2}{|c|}{ Post-Traumatic Stress Disorder } & $-0.239 *$ & $0.490 *$ & $-0.292 *$ & $-0.178^{*}$ & $-0.457^{*}$ & $-0.508 *$ \\
\hline \multicolumn{2}{|c|}{ General Self-Efficacy } & $0.440^{*}$ & $0.490 *$ & $0.446^{*}$ & $0.333^{*}$ & $0.447^{*}$ & $0.514 *$ \\
\hline \multirow{3}{*}{$\begin{array}{l}\text { Simplified Coping } \\
\text { Style }\end{array}$} & Total Score & $0.466 *$ & $0.419 *$ & $0.494^{*}$ & $0.297^{*}$ & $0.457^{*}$ & $0.508 *$ \\
\hline & Negative coping & $0.165 *$ & $0.068 *$ & $0.156^{*}$ & $0.043^{*}$ & $0.08 I^{*}$ & $0.138 *$ \\
\hline & Positive coping & $0.54 I^{*}$ & $0.542 *$ & $0.585^{*}$ & $0.388^{*}$ & $0.558^{*}$ & $0.618^{*}$ \\
\hline \multirow{4}{*}{$\begin{array}{l}\text { Social Support } \\
\text { Rate }\end{array}$} & Total Score & $0.424 *$ & $0.369 *$ & $0.468^{*}$ & $0.297^{*}$ & $0.394 *$ & $0.464 *$ \\
\hline & Utilization of support & $0.394 *$ & $0.319 *$ & $0.428^{*}$ & $0.246^{*}$ & $0.333^{*}$ & $0.4 I I *$ \\
\hline & Objective support & $0.238 *$ & $0.235^{*}$ & $0.282^{*}$ & $0.183^{*}$ & $0.242 *$ & $0.275^{*}$ \\
\hline & $\begin{array}{l}\text { Social Support Rate } \\
\text { Scale }\end{array}$ & $0.397^{*}$ & $0.336 *$ & $0.429 *$ & $0.269 *$ & $0.368^{*}$ & $0.432 *$ \\
\hline & & $\begin{array}{c}\text { New } \\
\text { possibilities }\end{array}$ & $\begin{array}{l}\text { Personal } \\
\text { strength }\end{array}$ & $\begin{array}{l}\text { Interpersonal } \\
\text { relationship }\end{array}$ & $\begin{array}{l}\text { Appreciate } \\
\text { life }\end{array}$ & $\begin{array}{l}\text { Mental } \\
\text { change }\end{array}$ & $\begin{array}{l}\text { Total } \\
\text { score }\end{array}$ \\
\hline
\end{tabular}

Note: $* p<0.05$.

Table 3 Multiple Regression Analysis of Influencing Factors of Nurses' PTG (A) and (B) Assignment Method of Independent Variable (A) Multiple Regression Analysis of Influencing Factors of Nurses' PTG

\begin{tabular}{|c|c|c|c|c|c|c|c|c|}
\hline & B $(95 \%$ Cl) & $\beta$ & $t$ & $P$ & $\mathbf{R}^{2}$ & $\Delta \mathbf{R}^{2}$ & $\mathbf{F}$ & $\mathbf{P}$ \\
\hline Model (constant) & 19.003 (14.903 to 23.102$)$ & - & 9.091 & $<0.001$ & 0.460 & & \multirow[t]{6}{*}{303.843} & \multirow[t]{6}{*}{$<0.001$} \\
\hline General self-efficacy & $0.800(0.697$ to 0.903$)$ & 0.298 & 15.234 & $<0.001$ & & 0.264 & & \\
\hline Coping style & $0.470(0.412$ to 0.528$)$ & 0.309 & 15.967 & $<0.001$ & & 0.112 & & \\
\hline Social support & $0.442(0.374$ to 0.510$)$ & 0.253 & 12.745 & $<0.001$ & & 0.067 & & \\
\hline PTSD & $-0.135(-0.178$ to -0.091$)$ & -0.114 & -6.067 & $<0.001$ & & 0.012 & & \\
\hline Professional title & $-1.161(-1.749$ to -0.573$)$ & -0.068 & -3.874 & $<0.001$ & & 0.005 & & \\
\hline$F$ & 303.843 & & & & & & & \\
\hline
\end{tabular}

(B) Assignment Method of Independent Variable

\begin{tabular}{|c|c|}
\hline Independent Variable & Assignment Way \\
\hline Highest degree & $\begin{aligned} \text { Technical secondary school } & =1 ; \text { junior college }=2 ; \text { undergraduate } 30 ; \text { Master's } \\
& \text { degree and higher }=4\end{aligned}$ \\
\hline Title & $\begin{aligned} \text { Nurse= }=\text {; Senior nurse = } & \text {; } \text { Nurse-in-charge }=3 ; \text { co-chief superintendent nurse =4; } \\
& \text { chief superintendent nurse }=5\end{aligned}$ \\
\hline Self-efficacy & Original value \\
\hline Coping strategy & Original value \\
\hline Social support & Original value \\
\hline Stress disorder & Original value \\
\hline
\end{tabular}

Note: $R^{2}=0.460$. 
accounting for a larger proportion. The conclusion of this study is consistent with the above view.

Clinical first-line nurses were more willing to adopt a positive coping style when facing the impact of COVID-19.

Self-efficacy refers to an individual's expectation or belief that they should be capable of taking measures to successfully implement and complete a particular behavior or goal or overcome a disadvantage. ${ }^{22}$ This study showed that the general self-efficacy of nurses was at a medium level, and self-efficacy could positively predict the level of PTG. Facing traumatic events would be regarded as a challenge and require a positive and optimistic attitude. Therefore, for people with low PTG, psychological adjustment could be carried out to improve their self-efficacy, thereby further improving their level of PTG.

\section{Negative Outcomes of PTG}

An interesting finding from this study was that the lower the nurse's professional title, the higher the PTG. This result is similar to the conclusion of Ma's research, ${ }^{24}$ the reason for which may be that nurses with low-level titles need more training, and frequent practice and assessment make them more confident to face the pandemic.

Another negative result was that PTSD symptoms was negatively related to PTG. Zebrack's research ${ }^{26}$ on young survivors reached a similar conclusion. Severe PTSD symptoms tended to predict lower levels of PTG. Conversely, frankly discussing fear, anxiety, and other emotions with family, friends, patients, or professionals could strengthen people's understanding of traumatic events and help them actively adapt to their future lives.

\section{Limitations}

The present study has some limitations. First, the use of self-reported instruments may have led patients to underreport or exaggerate the severity of their symptoms to minimize or exacerbate their problems. Second, we are aware that administering some of the questions in a retrospective way may have affected the answers provided by the patients due to memory biases. In addition, the cross-sectional design does not allow certain conclusions about causal direction to be drawn.

\section{Conclusion}

During the COVID-19 pandemic, clinical front-line nurses need to bear the burden of curing critically ill patients, and they also face the risk of contracting infectious diseases, so improving the mental health of nurses is a matter of urgency. The aim of this study was to investigate the current situation and factors related to the positive psychological effects on first-line nurses during the COVID-19 pandemic. It was found that good social support and self-efficacy are important factors in improving clinical nurses' PTG levels, and therefore nursing managers should pay attention to the negative emotions of clinical nurses; alleviate nurses' anxiety and fear through, for example, training and simulation exercises; and create a group atmosphere of mutual communication and help.

\section{Ethics Approval and Consent to Participate}

This study was conducted in accordance with the declaration of Helsinki. This study was conducted with approval from the Ethics Committee of Nantong First People's Hospital. A written informed consent was obtained from all participants.

\section{Disclosure}

All authors have contributed significantly to the manuscript and declare that the work is original and has not been submitted or published elsewhere. None of the authors have any financial disclosure or conflicts of interest in this work.

\section{References}

1. Heymann DL, Shindo N. COVID-19: what is next for public health? Lancet. $\quad 2020 ; 395(10224): 542-545 . \quad$ doi:10.1016/S0140-6736(20) 30374-3

2. Chinese Center for Disease Control and Prevention. Distribution of novel coronavirus as of 24:00 on April 24, 2020[EB/OL]. 2020. Available from: http://2019ncov.chinacdc.cn/2019-nCoV/global.html. Accessed April 24, 2020.

3. Bao Y, Sun Y, Meng S, Shi J, Lu L. 2019-nCoV epidemic: address mental health care to empower society. Lancet. 2020;395 (10224):37-38. doi:10.1016/S0140-6736(20)30309-3

4. Visser E, Gosens T, Den Oudsten BL, De Vries J. The course, prediction, and treatment of acute and posttraumatic stress in trauma patients: a systematic review. J Trauma Acute Care Surg. 2017;82 (6):1158-1183. doi:10.1097/TA.0000000000001447

5. Ripp J, Peccoralo L, Charney D. Attending to the emotional well-being of the health care workforce in a New York city health system during the COVID-19 pandemic. Acad Med. 2020;95 (8):1136-1139. doi:10.1097/ACM.0000000000003414

6. Di Tella M, Benfante A, Castelli L, Romeo A. Anxiety, depression, and posttraumatic stress in nurses during the COVID-19 outbreak. Intensive Crit Care Nurs. 2021;64:103014. doi:10.1016/j. iccn.2021.103014

7. Sampaio F, Sequeira C, Teixeira L. Nurses' mental health during the Covid-19 outbreak: a cross-sectional study. J Occup Environ Med. 2020;62(10):783-787. doi:10.1097/JOM.0000000000001987

8. Zoellner T, Maercker A. Posttraumatic growth in clinical psychology-A critical review and introduction of a two component model. Clin Psychol Rev. 2006;26(5):626-653. doi:10.1016/j. cpr.2006.01.008 
9. Wagner AC, Torbit L, Jenzer $\mathrm{T}$, et al. The role of posttraumatic growth in a randomized controlled trial of cognitive-behavioral conjoint therapy for PTSD symptoms. J Trauma Stress. 2016;29 (4):379-383. doi: $10.1002 /$ jts.22122

10. Ochoa C, Casellas-Grau A, Vives J, Font A, Borràs JM. Positive psychotherapy for distressed cancer survivors: posttraumatic growth facilitation reduces posttraumatic stress. Int J Clin Health Psychol. 2017;17(1):28-37. doi:10.1016/j.ijchp.2016.09.002

11. Wang L, Li X, Li Y, Li L. Status quo of reproductive concerns in patients with breast cancer receiving postoperative chemotherapy and its influencing factors. Chin J Mod Nurs. 2019;25(1):58-62.

12. Marziliano A, Tuman M, Moyer A. The relationship between post-traumatic stress and post-traumatic growth in cancer patients and survivors: a systematic review and meta-analysis. Psychooncology. 2020;29(4):604-616. doi:10.1002/pon.5314

13. Inan FŞ, Üstün B. Post-traumatic growth in the early survival phase: from Turkish breast cancer survivors' perspective. Eur J Breast Health. 2019;16(1):66-71. doi:10.5152/ejbh.2019.5006

14. Zhang L, Lu Y, Qin Y, Xue J, Chen Y. Post-traumatic growth and related factors among 1221 Chinese cancer survivors. Psychooncology. 2020;29(2):413-422. doi:10.1002/pon.5279

15. Tedeschi RG, Calhoun LG. The posttraumatic growth inventory: measuring the positive legacy of trauma. J Trauma Stress. 1996;9 (3):455-471. doi:10.1002/jts.2490090305

16. Wang J, Wang YB, Liu XH. Research progress of tools for measuring post-traumatic growth. Chin J Nurs. 2010;45(8):758-760.

17. Weathers FW, Litz BT, Herman DS, Huska JA, Keane TM. The PTSD symptoms checklist: reliability, validity, and diagnostic utility. Int Soc Trauma Stress Stud. 1993;2:90-92.

18. Tarsitani L, Vassalini P, Koukopoulos A, et al. Post-traumatic stress disorder among COVID-19 survivors at 3-month follow-up after hospital discharge. J Gen Intern Med. 2021;1-6. doi:10.1007/ s11606-020-05956-2
19. Wulfes N, Del Pozo MA, Buhr-Riehm B, Heinrichs N, Kröger C. Screening for posttraumatic stress disorder in refugees: comparison of the diagnostic efficiency of two self-rating measures of posttraumatic stress disorder. J Trauma Stress. 2019;32(1):148-155. doi: $10.1002 /$ jts. 22358

20. Xie YN. A preliminary study on the reliability and validity of the simple coping style scale. Chin J Clin Psychol. 1998;6(2):114-115.

21. Shao R, He P, Ling B, et al. Prevalence of depression and anxiety and correlations between depression, anxiety, family functioning, social support and coping styles among Chinese medical students. $B M C$ Psychol. 2020;8(1):38. doi:10.1186/s40359-020-00402-8

22. Schwarzer R, Artisti B. Optimistic self-beliefs: assessment of general perceived self-efficacy in thirteen cultures. Wordpsychol. 1997;3 (1-2):177-190

23. Cheung SK, Sun SY. Assessment of optimistic self-beliefs: further validation of the Chinese version of the general self-efficacy scale. Psychol Rep. 1999;85(3 Pt 2):1221-1224. doi:10.2466/ pr0.1999.85.3f.1221

24. Ma XJ, Chen LL, Yang JL, Zhou C. Post traumatic growth and its influencing factors in emergency nurses. Chin J Mod Nurs. 2018;24 (030):3655-3658.

25. Hu R, Wang X, Liu Z, et al. Stigma, depression, and post-traumatic growth among Chinese stroke survivors: a longitudinal study examining patterns and correlations. Top Stroke Rehabil. 2020;12 (28):1-14. doi:10.1080/10749357.2020.1864965

26. Zebrack B, Kwak M, Salsman J, et al. The relationship between posttraumatic stress and posttraumatic growth among adolescent and young adult (AYA) cancer patients. Psycho-Oncology. 2015;24 (2):162-168. doi:10.1002/pon.3585
Risk Management and Healthcare Policy

\section{Publish your work in this journal}

Risk Management and Healthcare Policy is an international, peerreviewed, open access journal focusing on all aspects of public health, policy, and preventative measures to promote good health and improve morbidity and mortality in the population. The journal welcomes submitted papers covering original research, basic science, clinical \& epidemiological studies, reviews and evaluations, guidelines, expert opinion and commentary, case reports and extended reports. The manuscript management system is completely online and includes a very quick and fair peer-review system, which is all easy to use. Visit http://www.dovepress.com/testimonials.php to read real quotes from published authors. 